\title{
Efeito acaricida do óleo essencial de Melaleuca alternifolia sobre Otodectes cynotis
}

\author{
Acaricide effect of the Melaleuca alternifolia essential oil \\ on Otodectes cynotis
}

\author{
Rita de Cássia da Silva Machado Neves, ${ }^{*}$ Rosa Helena dos Santos Ferraz, ${ }^{* *}$ Adriane Jorge Mendonça, ${ }^{* *}$ \\ Samara Rosolem Lima, ${ }^{* *}$ Felipe Augusto Constantino Seabra da Cruz, ${ }^{* *}$ Jaqueline Gomes Rosa, ${ }^{* *}$ \\ Lúcia Aparecida de Fátima Mateus, ${ }^{* * *}$ Vany Ferraz, ${ }^{* * *}$ Luciano Antunes Barros ${ }^{* * * *}$
}

\begin{abstract}
Resumo
A otocariose é uma doença parasitária causada pelo ácaro Otodectes cynotis, sendo frequentemente observado no ouvido de cães e gatos, como um dos principais causadores de otite externa. Para o tratamento da otocariose é necessário o uso de substâncias acaricidas. No entanto, é crescente o número de relatos sobre a resistência parasitária, sendo este um motivo de preocupação por parte de clínicos e proprietários. Com o objetivo de avaliar o efeito acaricida do óleo essencial de Melaleuca alternifolia (tea tree) sobre a sarna Otodectes cynotis, foram formados três grupos experimentais de dez animais cada, que receberam os seguintes tratamentos: Grupo um: loção a 5\% de óleo essencial de M. alternifolia; Grupo dois: loção a 5\% de tiabendazol; Grupo três: loção à base de uma cera emulsificante não iônica. Todos os animais tiveram seus condutos auditivos externos tratados durante sete dias consecutivos e examinados periodicamente a fim de se observar a eficácia dos produtos. Concluiu-se que o óleo essencial de $M$. alternifolia pode ser empregado na espécie canina, como forma de tratamento para otite por O. cynotis.
\end{abstract}

Palavras-chave: Otodectes cynotis, otocariose, Melaleuca alternifolia, teste in vivo.

\begin{abstract}
The otocariose is a parasitic disease caused by mite Otodectes cynotis, frequently observed in the ear of dogs and cats as a major cause of otitis externa. For treatment of otocariose need substance use acaricides. However an increasing number of reports of parasite resistance, which is a cause for concern on the part of veterinarys and owners. With the aim of evaluating the acaricide effect of the Melaleuca alternifolia essential oil (tea tree) on the Otodectes cynotis scabies, three experimental groups of ten animals were formed, that received the following treatments: Group one: $5 \%$ M. alternifolia essential oil lotion; Group two: $5 \%$ of tiabendazol lotion; Group three: non-ionic emulsifying wax-based lotion. All the animals had their external auditory canals treated during seven days and periodically examined in order to observe the efficacy of the products. It is concluded that the M. alternifolia essential oil can be used in dogs, as treatment for otitis caused by O. cynotis.
\end{abstract}

Keywords: Otodectes cynotis, otocariosis, Melaleuca alternifolia, in vivo test.

\section{Introdução}

As otites caninas produzem grande desconforto físico para os pacientes e constituem um dos principais motivos de consultas a médicos-veterinários, representando cerca de 8 a $15 \%$ dos casos atendidos na prática clínica veterinária no Brasil (Leite, 2000).

A classificação desta patologia vai variar conforme a área afetada do conduto auditivo. Assim, as otites se classificam em internas, médias e externas.

A otite externa geralmente é definida como a inflamação do canal auditivo e pode também envolver a porção mais externa do pavilhão auricular. Os sinais clínicos associados à otite externa incluem graus variados de sacudidas da cabeça, prurido, dor, odor e exsudação.
Estima-se que em torno de 5 a $20 \%$ dos cães apresentam otite externa (August, 1988), sendo sua prevalência ainda maior em regiões que apresentam clima tropical, provavelmente próxima a 30 ou $40 \%$ (Logas, 1994). Na forma externa, a otite canina pode ser determinada por uma combinação de fatores predisponentes, primários e perpetuadores (August, 1988; Logas, 1994; Scott et al., 1996)1996.

Dentre os fatores primários, destaca-se o ácaro Otodectes cynotis, que parasita frequentemente o ouvido de cães e gatos, atuando como um dos principais causadores de otite externa (Six et al., 2000; Lohse et al., 2002).

Ao se planejar um protocolo terapêutico para otite externa, deve-se considerar um possível efeito ototóxico da substância a ser utilizada. Um agente ototóxico é definido como qualquer

\footnotetext{
* Hospital Veterinário, Universidade Federal de Mato Grosso. Autor para correspondência. E-mail: nevesrita@uol.com.br.

** Faculdade de Agronomia, Medicina Veterinária e Zootecnia, Universidade Federal de Mato Grosso.

*** Faculdade de Biociências, Universidade Federal de Mato Grosso.

**** Departamento de Química, Universidade Federal de Minas Gerais.

${ }^{* * * * *}$ Faculdade de Veterinária, Universidade Federal Fluminense.
} 
substância que possa induzir à perda da audição ou à ocorrência de distúrbios do equilíbrio, devido às lesões das estruturas das orelhas médias e/ou internas (Merchant, 1994). Os medicamentos tópicos podem também causar efeitos sistêmicos, principalmente quando houver perfuração timpânica e/ou ulcerações na parede do conduto auditivo (Nuttall e Cole, 2004; Tuleski, 2007).

O uso de um acaricida é essencial para o tratamento da otocariose, existindo a indicação do tiabendazol e do diazinon, como drogas otológicas disponíveis com efeito residual limitado, requerendo aplicação regular por no mínimo 10 dias até se completar o ciclo do ácaro, uma vez que estas substâncias não apresentam efeito sobre os ovos (Curtis, 2004). Há um crescente número de registros sobre resistência contra compostos antiectoparasitos. Relatos sobre os insucessos do tratamento da escabiose (Sarcoptes scabiei) com lindano, crotamiton e benzoato de benzila, bem como a provável resistência emergente para $5 \%$ de permetrina e ivermectina oral, são motivos que justificam a busca por novas drogas acaricidas (Walton et al., 2004).

Nas últimas décadas, estudos intensivos têm avaliado plantas como fonte de produtos efetivos para a conservação da saúde humana (Bertini et al., 2005). Os óleos essenciais são misturas complexas de substâncias, geralmente odoríferas e líquidas, sendo sua principal característica a volatilidade. Em sua grande maioria, estes óleos são de aroma agradável e intenso, sendo chamados de óleos essências (Jesus et al., 2007).

A Melaleuca alternifolia, ou tea tree (árvore do chá), como é conhecida popularmente, é nativa da Austrália e seu óleo essencial possui comprovada ação antimicrobiana contra bactérias, fungos, alguns vírus além de forte atividade repelente contra mosquitos, pulgas e piolhos (Silva et al., 2002). Seus principais componentes ativos são os terpenoides oxigenados (Walton et al., 2004). Dentre muitos, existem dois significantes: o 1,8 cineol e o terpinen-4-ol, com níveis máximos e mínimos estabelecidos para ambos, principalmente porque o cineol é um conhecido irritante da pele e o terpinen-4-ol é apontado como o maior contribuinte da atividade antimicrobiana dentre os componentes (Simões et al., 2002).

Resultados de estudos com outros ácaros sugerem que o óleo essencial de M.alternifolia apresenta um efeito evidente como um novo parasiticida tópico (Walton et al., 2004).

Desta forma, o objetivo deste estudo foi avaliar o efeito acaricida, in vivo, do óleo essencial de Melaleuca sobre o ácaro O. cynotis parasitando cães.

\section{Material e métodos}

Foram utilizados 30 cães provenientes de um abrigo particular, localizado no município de Cuiabá e diagnosticados como positivos para a infestação pelo ácaro $O$. cynotis, através da otoscopia.

Por se tratar de um ensaio clínico, o critério para seleção dos animais foi apenas o de positividade, independentemente de porte, sexo, raça, tipo de orelha ou idade. Assim, os cães utilizados no experimento foram 15 (50\%) de pequeno porte, 12 (40\%) de médio porte e três (10\%) de grande porte. Em relação ao sexo, 19 (63\%) eram fêmeas e 11 (37\%) eram machos. Quanto à raça dos animais, 13 (43\%) eram de raça (um boxer; dois cockers americanos; oito cockers ingleses; dois poodles) e $17(57 \%)$ sem raça definida. Em se tratando da posição das orelhas, um $(3,3 \%)$ animal tinha orelha ereta, $25(83,3 \%)$ de forma pendular e quatro $(13,3 \%)$ de forma semiereta. Todos os animais eram adultos. Considerou-se cada orelha como uma unidade experimental.

Os cães foram distribuídos aleatoriamente, por método de sorteio, em três grupos de acordo com os produtos a serem testados, sendo, portanto, cada grupo constituído por 10 animais.

Grupo um: animais tratados com loção otológica a $5 \%$ de óleo essencial de M. alternifolia;

Grupo dois: animais tratados com loção otológica a $5 \%$ de tiabendazol;

Grupo três: animais tratados com o veículo das loções anteriores, cera emulsificante não iônica denominada Polawax®, utilizada na formulação de bases farmacológicas (Catunda et al., 2007).

Foram utilizadas para identificação dos animais, coleiras de cores diferentes, adotando-se uma cor para cada um dos três grupos. Os animais foram mantidos em três canis separados, respeitando a distribuição por grupo, não sendo aplicado nenhum tipo de tratamento no corpo ou no ambiente durante o período do experimento.

Os cães foram examinados, apenas por otoscopia, três dias antes do tratamento (dia -3) e o primeiro dia do tratamento foi considerado como dia 0 (zero).

O tratamento consistiu da aplicação de um $\mathrm{mL}$ de solução otológica em cada conduto auditivo, uma vez ao dia, por sete dias consecutivos. Esta aplicação foi feita com seringa descartável, de um $\mathrm{mL}$, estéril, descartada após o único uso em um dos condutos auditivos.

A qualidade comercial do óleo essencial de $M$. alternifolia é determinada pelas concentrações dos compostos terpinen-4-ol, e 1,8-cineol, que devem ser de no mínimo $30 \%$ e no máximo $15 \%$ respectivamente (Silva et al., 2002). O óleo essencial de M. alternifolia utilizado foi manipulado, para confecção da loção, sendo analisado, quimicamente, pelo método de cromatografia gasosa, no Laboratório de Cromatografia, Departamento de Química da UFMG, atestando-se que o produto encontrava-se dentro dos padrões.

Os animais foram reexaminados, por otoscopia, nos dias $+1,+3$, $+7,+10,+17,+24,+31$ para verificação da eficácia dos produtos e de possíveis reinfestações. Não foi realizada limpeza, nem retirada de secreção dos condutos auditivos antes ou durante o período de observação. Ainda no dia +31 , após a otoscopia, foi coletada secreção auricular do canal vertical, bilateralmente, com auxílio do swab, fazendo-se a introdução deste instrumento no canal e posterior rotação de $360^{\circ}$ no sentido horário.

Imediatamente após cada coleta, por meio de visualização, em microscópio estereoscópico, observou-se o swab parasitológico à procura de ácaros, a fim de se confirmar o diagnóstico positivo ou negativo.

Provas bioquímicas do sangue tiveram a finalidade de detectar possíveis alterações sistêmicas como função hepática (Alanina Amino Transferase - ALT e Fosfatase Alcalina - FA) e renal (Ureia e Creatinina) dos animais antes e após o tratamento. Assim, foram colhidos sete $\mathrm{ml}$ de sangue, de cada animal, imediatamente antes da aplicação dos produtos e no dia imediatamente posterior ao último dia de tratamento. 
Imediatamente após o término do experimento, todos os animais positivos integrantes do grupo controle e todos os outros animais do abrigo diagnosticados como positivos, foram tratados com loção otológica a 5\% de óleo essencial de M. alternifolia.

A avaliação da eficácia do produto, em cada orelha, foi realizada segundo a fórmula: ( $\mathrm{N}^{\circ}$ de orelhas infestadas antes do tratamento - $\mathrm{N}^{\circ}$ de orelhas infestadas após o tratamento) / $\mathrm{n}^{\circ}$ de orelhas infestadas antes do tratamento $X 100$ (Souza et al., 2006).

Este estudo foi aprovado pelo Comitê de Ética em Pesquisa Animal da UFMT, protocolo $n^{\circ} 23108.030616 / 09-3$, estando de acordo com os Princípios Éticos na Experimentação Animal.

\section{Resultados}

Observou-se, no grupo um (com óleo essencial de $M$. alternifolia), o percentual médio de eficácia do produto variando entre $90 \%$, em relação à orelha direita, e $100 \%$, para a orelha esquerda, no $1^{\circ}$ dia após o início do tratamento. Nos dias +3 e +7 a eficácia do produto, em ambas as orelhas, se manteve em $100 \%$. No dia +10 a eficácia foi de $100 \%$, em relação à orelha direita e de $90 \%$ para a orelha esquerda. Este último percentual se manteve, em ambas as orelhas, do dia +24 ao +31 . O dia 17 apresentou eficácia de $100 \%$, em ambas as orelhas. Estes resultados estão apresentados na Tabela 1 .

Tabela 1: Acompanhamento otoscópico antes (dia - 3), durante (dias 0 a +7) e após (dias $+10 \mathrm{a}+31$ ) o tratamento dos condutos auditivos com loção de melaleuca a $5 \%$ e diagnóstico final pelo swab parasitológico $($ dia +31$)$

\begin{tabular}{|c|c|c|c|c|c|c|c|c|c|c|c|c|c|c|c|c|c|c|}
\hline \multirow{4}{*}{ Cães } & \multicolumn{18}{|c|}{ Dias } \\
\hline & \multicolumn{16}{|c|}{ Otoscopia } & \multirow{2}{*}{\multicolumn{2}{|c|}{$\begin{array}{c}\text { Swab } \\
+31\end{array}$}} \\
\hline & \multicolumn{2}{|c|}{-3} & \multicolumn{2}{|c|}{+1} & \multicolumn{2}{|c|}{+3} & \multicolumn{2}{|c|}{+7} & \multicolumn{2}{|c|}{+10} & \multicolumn{2}{|c|}{+17} & \multicolumn{2}{|c|}{+24} & \multicolumn{2}{|c|}{+31} & & \\
\hline & D & $E$ & D & $E$ & D & $E$ & D & $E$ & D & $E$ & & $E$ & D & $E$ & D & $E$ & D & $E$ \\
\hline 1 & + & + & - & - & - & - & - & - & - & + & - & - & + & + & + & + & + & + \\
\hline 2 & + & + & + & - & - & - & - & - & - & - & - & - & - & - & - & - & - & - \\
\hline 3 & + & + & - & - & - & - & - & - & - & - & - & - & - & - & - & - & - & - \\
\hline 4 & + & + & - & - & - & - & - & - & - & - & - & - & - & - & - & - & - & - \\
\hline 5 & + & + & - & - & - & - & - & - & - & - & - & - & - & - & - & - & - & - \\
\hline 6 & + & + & - & - & - & - & - & - & - & - & - & - & - & - & - & - & - & - \\
\hline 7 & + & + & - & - & - & - & - & - & - & - & - & - & - & - & - & - & - & - \\
\hline 8 & + & + & - & - & - & - & - & - & - & - & - & - & - & - & - & - & - & - \\
\hline 9 & + & + & - & - & - & - & - & - & - & - & - & - & - & - & - & - & - & - \\
\hline 10 & + & + & - & - & - & - & - & - & - & - & - & - & - & - & - & - & - & - \\
\hline
\end{tabular}

$\mathrm{D}=$ conduto auditivo direito

$\mathrm{E}=$ conduto auditivo esquerdo

Não foram observadas reações otológicas adversas, relacionadas com o tratamento, durante o período de experimentação nem alterações nas funções hepáticas e renais dos animais.

Observou-se, no grupo dois (com tiabendazol), o percentual médio de eficácia do produto variando entre zero para a orelha esquerda, e $20 \%$, para a orelha direita, no $1^{\circ}$ dia após o início do tratamento. No dia +3 , a eficácia do produto variou entre 80 e $90 \%$ para as orelhas direita e esquerda, respectivamente. Do dia +7 ao +31 esta eficácia foi de $100 \%$, em ambas as orelhas, considerando-se a observação pela otoscopia. Porém, no exame do swab parasitológico, uma das orelhas mostrou-se positiva, reduzindo a eficácia do produto na orelha esquerda para $90 \%$. Estes resultados estão apresentados na Tabela 2.
No grupo três (controle), a eficácia do produto se manteve nula do dia +1 ao +3 após o tratamento. Levando-se em consideração que cada orelha corresponde a uma unidade experimental, observa-se no dia +7 o percentual médio de eficácia do produto variando entre $10 \%$ em relação à orelha direita e $20 \%$ à orelha esquerda. Entre os dias +10 a +17 a eficácia do produto se manteve em $10 \%$, tornando a ocorrer um percentual médio semelhante em relação à orelha esquerda no dia +24 . No restante dos dias da otoscopia, foi possível observar ácaros vivos circulando nos 20 condutos auditivos e, sobre o cerúmen, na observação ao microscópio estereoscópico. Estes resultados estão apresentados na Tabela 3.

O percentual de eficácia, calculado para cada produto, nas determinadas horas, permitiu uma análise descritiva de cada um e a comparação entre ele.

\section{Discussão}

Embora este seja o primeiro relato do uso do óleo essencial de M. alternifolia no tratamento da otocariose, as eficácias aqui atingidas são compatíveis às descritas no estudo, in vitro, de Walton et al. (2004), com Sarcoptes scabiei var. hominis. No referido estudo, o óleo essencial de melaleuca a $5 \%$ e seu componente ativo, terpinen-4-ol, foram altamente eficazes na redução do tempo de sobrevivência do parasito. Ainda, em outra pesquisa, in vitro, Gao et al. (2005), obtiveram a morte do ácaro Demodex folliculorum em 2 horas e 30 minutos e uma redução drástica, in vivo, em grande parte dos pacientes.

Ramacciato (2000) em um estudo odontológico in vivo, utilizando solução de óleo de M.alternifolia a $0,2 \%$, observou efeito residual de duas semanas póstratamento para micro-organismos totais e Streptococcus mutans. Gao et al. (2005) relataram que o óleo essencial de M.alternifolia tem a capacidade de matar piolhos, mediada pela atividade anticolinesterase. Na presente pesquisa, fica a dúvida sobre o poder residual e/ ou sobre a capacidade de M.alternifolia contra os ovos dos ácaros, já que um dos animais tratados apresentou-se resultado positivo a partir do dia +24 , mantendose, assim, até o fim do experimento. Entretanto, deve-se esclarecer que o animal que se manteve positivo era um cão com sequelas de cinomose, com sinais neurológicos e paralisia dos membros pélvicos, permanecendo por quase todo o tempo com grande parte do corpo em contato com o piso, facilitando, desta forma, a reinfestação. Esta hipótese está de acordo com as observações de Souza (2004), que indica a influência do piso cimentado como abrigo importante para os ácaros. Mais um motivo para, ainda, justificar a parasitose neste cão é o fato dele apresentar orelhas pendentes, cujo formato restringe a entrada de luz no conduto auditivo, acarretando maior umidade local, circunstância essa propícia à multiplicação do ácaro, estando de acordo com Mccallum Jr. (1967).

Nenhum cão apresentou efeitos colaterais, sistêmicos ou dermatológicos. Gao et al. (2005) acreditam que o fato de não 
Tabela 2: Acompanhamento otoscópico antes (dia - 3), durante (dias 0 a +7) e após (dias $+10 \mathrm{a}+31$ ) o tratamento dos condutos auditivos com loção de tiabendazol a $5 \%$ e diagnóstico final pelo swab parasitológico $(\mathrm{dia}+31)$

\begin{tabular}{|c|c|c|c|c|c|c|c|c|c|c|c|c|c|c|c|c|c|c|}
\hline \multirow{4}{*}{ Cães } & \multicolumn{18}{|c|}{ Dias } \\
\hline & \multicolumn{16}{|c|}{ Otoscopia } & \multirow{2}{*}{\multicolumn{2}{|c|}{$\begin{array}{c}\text { Swab } \\
+31\end{array}$}} \\
\hline & \multicolumn{2}{|c|}{-3} & \multicolumn{2}{|c|}{+1} & \multicolumn{2}{|c|}{+3} & \multicolumn{2}{|c|}{+7} & \multicolumn{2}{|c|}{+10} & \multicolumn{2}{|c|}{+17} & \multicolumn{2}{|c|}{+24} & \multicolumn{2}{|c|}{+31} & & \\
\hline & D & $E$ & D & $\mathbf{E}$ & D & $E$ & D & $E$ & D & $E$ & D & $\mathbf{E}$ & D & $\mathbf{E}$ & D & $E$ & D & $E$ \\
\hline 1 & + & + & + & + & - & - & - & - & - & - & - & - & - & - & - & - & - & - \\
\hline 2 & + & + & + & + & + & - & - & - & - & - & - & - & - & - & - & - & - & - \\
\hline 3 & + & + & + & + & + & + & - & - & - & - & - & - & - & - & - & - & - & - \\
\hline 4 & + & + & + & + & - & - & - & - & - & - & - & - & - & - & - & - & - & - \\
\hline 5 & + & + & + & + & - & - & - & - & - & - & - & - & - & - & - & - & - & - \\
\hline 6 & + & + & + & + & - & - & - & - & - & - & - & - & - & - & - & - & - & - \\
\hline 7 & + & + & - & + & - & - & - & - & - & - & - & - & - & - & - & - & - & - \\
\hline 8 & + & + & - & + & - & - & - & - & - & - & - & - & - & - & - & - & - & - \\
\hline 9 & + & + & + & + & - & - & - & - & - & - & - & - & - & - & - & - & - & - \\
\hline 10 & + & + & + & + & - & - & - & - & - & - & - & - & - & - & - & - & - & + \\
\hline
\end{tabular}

$\mathrm{D}=$ conduto auditivo direito

$\mathrm{E}=$ conduto auditivo esquerdo

Tabela 3: Acompanhamento otoscópico antes (dia - 3), durante (dias 0 a +7) e após (dias $+10 a+31)$ o tratamento dos condutos auditivos com loção veículo e diagnóstico final pelo swab parasitológico $(\mathrm{dia}+31)$

\begin{tabular}{|c|c|c|c|c|c|c|c|c|c|c|c|c|c|c|c|c|c|c|}
\hline \multirow{4}{*}{ Cães } & \multicolumn{18}{|c|}{ Dias } \\
\hline & \multicolumn{16}{|c|}{ Otoscopia } & \multirow{2}{*}{\multicolumn{2}{|c|}{$\begin{array}{c}\text { Swab } \\
+31\end{array}$}} \\
\hline & \multicolumn{2}{|c|}{-3} & \multicolumn{2}{|c|}{+1} & \multicolumn{2}{|c|}{+3} & \multicolumn{2}{|c|}{+7} & \multicolumn{2}{|c|}{+10} & \multicolumn{2}{|c|}{+17} & \multicolumn{2}{|c|}{+24} & \multicolumn{2}{|c|}{+31} & & \\
\hline & D & $E$ & D & $E$ & D & $E$ & D & $E$ & D & $E$ & D & $E$ & D & $E$ & D & $E$ & D & $E$ \\
\hline 1 & + & + & + & + & + & + & + & + & + & + & + & + & + & - & + & + & + & + \\
\hline 2 & + & + & + & + & + & + & + & + & + & + & + & + & + & + & + & + & + & + \\
\hline 3 & + & + & + & + & + & + & + & - & + & + & + & - & + & + & + & + & + & + \\
\hline 4 & + & + & + & + & + & + & - & + & + & + & - & + & + & + & + & + & + & + \\
\hline 5 & + & + & + & + & + & + & + & + & + & + & + & + & + & + & + & + & + & + \\
\hline 6 & + & + & + & + & + & + & + & + & + & + & + & + & + & + & + & + & + & + \\
\hline 7 & + & + & + & + & + & + & + & - & + & - & + & + & + & + & + & + & + & + \\
\hline 8 & + & + & + & + & + & + & + & + & - & + & + & + & + & + & + & + & + & + \\
\hline 9 & + & + & + & + & + & + & + & + & + & + & + & + & + & + & + & + & + & + \\
\hline 10 & + & + & + & + & + & + & + & + & + & + & + & + & + & + & + & + & + & + \\
\hline
\end{tabular}

$\mathrm{D}=$ conduto auditivo direito

$\mathrm{E}=$ conduto auditivo esquerdo

se detectar reações adversas, seja devido ao fato da DL50 dérmica ser superior a $5 \mathrm{~g} / \mathrm{kg}$ em coelhos e do óleo essencial de $M$. alternifolia puro não produzir efeitos fototóxicos na pele dos camundongos, possibilitando neste estudo o uso do referido óleo a $5 \%$ com bastante segurança.

Quanto ao tiabendazol, os achados deste estudo não são compatíveis com os obtidos por outros autores, pois no dia +1 a eficácia ficou entre zero e $20 \%$. Faulk e Schwirck (1978), obtiveram a eficácia de 94,4 e 100\% em cães e gatos respectivamente e Souza et al. (2006) obtiveram um efeito letal de 20 e $80 \%$, já no primeiro dia do experimento. Esta diferença de eficácia do tiabendazol pode ter ocorrido em função da origem da matéria-prima para a fabricação da loção. No trabalho de Souza et al. (2006) o produto em questão tinha como origem a indústria farmacêutica nacional - Foldan ${ }^{\circledR}$ e, no presente estudo, embora as bases fossem iguais e na mesma concentração, esta foi produzida em farmácia de manipulação, pois se desejava usar os mesmos veículos dos outros dois produtos manipulados no experimento, podendo existir diferença na qualidade do produto utilizado.

Quanto ao poder residual, no presente trabalho, uma das orelhas de um animal foi positiva ao exame de swab parasitológico, no último dia do experimento. No estudo in vivo de Patterson e Kirchain (1999), com furões, houve $60 \%$ de eficácia na quarta semana, $80 \%$ na sexta e $100 \%$ na sétima, ocorrendo reinfestação na oitava semana. Vale ressaltar que Chester (1988) e Harvey et al. (2004) afirmaram que o tiabendazol possui propriedade acaricida, matando o ácaro em todos os estágios de vida. Entretanto, Curtis (2004) relata baixo poder residual e a necessidade de reaplicações frequentes, por alegar que o produto não atinge os ovos.

Analisando-se os resultados de forma descritiva e comparando-se o grupo de animais tratados com óleo essencial de $M$. alternifolia com o grupo tratado com tiabendazol, observa-se uma excelente ação acaricida para ambos os tratamentos; no entanto, no dia +1 , foi evidenciada uma melhor ação acaricida no grupo submetido ao tratamento com $M$. alternifolia.

A aplicação da loção controle não foi capaz de minimizar a infestação por O. cynotis, servindo, de forma efetiva neste experimento como um produto inerte.

\section{Conclusão}

Foi possível concluir que o óleo de $M$. alternifolia, a $5 \%$, possui propriedade acaricida, in vivo, sobre ácaros do gênero 0 . cynotis, sem reações adversas, sendo, assim, mais uma opção para o médico-veterinário, no tratamento da otocariose canina. 


\section{Referências}

AUGUST, J. Otitis externa. A disease of multifactorial etiology. Vet. Clin North Am Small Anim Pract., v. 18, n. 4, p. 731-742, 1988.

BERTINI, L.M.; PEREIRA, A.F.; OLIVEIRA, C.L.L.; MENEZES, E.A.; MORAIS, S.M.; CUNHA, F.A.; BARREIRA, E.S.C. Perfil da Sensibilidade de Bactérias Frente a Óleos Essenciais de Algumas Plantas do Nordeste do Brasil. Inf, v. 17, n. 3/4, p. 80-83, 2005.

CATUNDA, A. P. M.; NEVES, I. P. F.; MEDEIROS, M. A. P.; MIRANDA, N. A.; SILVEIRA, W. L. L.; ARAGAO, C. F. S. Avaliação do comportamento de diferentes lotes do creme Polawax ${ }^{\circledR}$ comercializados na cidade de Natal/RN. In: XLVII CONGRESSO BRASILEIRO DE QUÍMICA, 9., 2007, Natal. Anais eletrônicos do Congresso Brasileiro de Química. Natal: ABQ, 2007. Disponível em:<http://www.abq.org.br/cbq/2007/trabalhos/4/4-467-640.htm>. Acesso em: 20 dez. 2009.

CHESTER, D. Medical management of otitis externa. Vet. Clin North Am Small Anim Pract., v. 18, n. 4, p. 799-812, 1988.

CURTIS, C. Current trends in the treatment of Sarcoptes, Cheyletiella and Otodectes mite infestations in dogs and cats. Vet. Dermatol., v. 15, n. 2, p. 108-114, 2004.

FAULK, R.; SCHWIRCK, S. Effect of Tresaderm against otoacariasis: a clinical trial. Vet. Med Small Anim Clin., v. 73, n. 3, p. 307-308, 1978.

GAO, Y.; DI PASCUALE, M.A.; BARADARAN-RAFI, A.; ELIZONDO, A.; KUO, C-L.; RAJU, V.K.; TSENG, S.C.G. In vitro and in vivo killing of ocular Demodex by tea tree oil. Br. J Ophthalmol., v. 89, n. 11, p. 1468-1473, 2005.

HARVEY, R.G.; HARARI, J.; DELAUCHE, A.J. Doença do Ouvido em Cães e Gatos. Rio de Janeiro: Revinter, 2004.

JESUS, E.R.; BARIN, C.S.; MACHADO, R.E. Óleo Essencial de Melaleuca alternifolia - Otimização do Método de Análise. XV ENCONTRO DE QUIMICA DA REGIAO SUL. UEPG. 2007. Arapongas-PR

LEITE, C.A.L. As otites de cães e gatos. Parte 1 - Epidemiologia. Cães e Gatos, v. 15, p. 22-26, 2000.

LOGAS, D.B. Diseases of the Ear Canal. Vet. Cli of North Am Small Anim Pract., v. 24, n. 5, p. 905-919, 1994.

LOHSE, J.; RINDER, H.; ZAHLER, M. Validity of species status of the parasitic mite otodectes cynotis. Med. Vet Entomol., v. 16, n. 2, p. 133-138, 2002.

MCCALLUM Jr., P.P. Inapparent infestation of Otodectes cynotis in Dog and Cat. Georg. Vet., v. 19, p. 8-9, 1967.

MERCHANT, S.R. Ototoxicity. Vet. Cli North Am Small Anim Pract., v. 24, n. 5, p. 971-980, 1994.
NUTTALL, T.; COLE, L. Ear cleaning: the UK and US perspective. Vet. Dermatol., v.15, n. 2, p.127-136, 2004.

PATTERSON, M.M.; KIRCHAIN, S.M. Comparasion of Three Treatments for Control of Ear Mites in Ferrets. Am. Assoc Lab Anim Science., v. 49, n. 6, p. 655-657, 1999.

RAMACCIATO, J.C. Atividade Antimicrobiana de Soluções à Base de Alho (Allium sativum), Óleo de Melaleuca (Melaleuca alternifolia) e Clorexidina Sobre Microorganismos Totais e Estreptococos do Grupo Mutans. Estudo in Vivo. 2000. 88 f. Disertação (Mestrado) - Faculdade de Odontologia de Piracicaba, Universidade Estadual de Campinas, Piracicaba, 2000.

SCOTT, D.W.; MILLER, W.H.; GRIFFIN, C.E. Muller e kirk. Dermatologia de Pequenos Animais. 5. ed. Rio de Janeiro: Interlivros, 1996.

SILVA, R.S.S.; DEMUNER, A.J.; BARBOSA, L.A.; CASALI, V.W.D.; NASCIMENTO, E.A.; PINHEIRO, A.L. Efeito do Estresse Hídrico sobre Características de Crescimento e a Produção de Óleo Essencial de Melaleuca alternifolia Cheel. Acta. Sci., v. 24, n. 5, p. 363-368, 2002

SIMÕES, R.P.; GROPPO, F.C.; SARTORATO, A.; DEL FIOL, F.S FILHO, T.R.M.; RAMACCIATO, J.C.; RODRIGUES, M.V.N. Efeito do Óleo de Melaleuca alternifolia sobre a Infecção Estafilócica. Rev Lecta., n. 2, p. 143-152, 2002.

SIX, R.H.; CLEMENCE, R.G.; THOMAS, C.A.; BEHAN, S.; BOY, M.G.; WATSON, P.; BENCHAOUI, H.A.; CLEMENTS, P.J.; ROWAN T.G.; JERNIGAN, A.D. Efficacy and safety of selamectin against Sarcoptes scabiei on dogs and Otodectes cynotis on dogs and cats presented as veterinary patients. Vet. Parasitol., v. 91, n. 3-4, p. 291-309, 2000.

SOUZA, C.P. Otite parasitária por Otodectes cynotis (Hering, 1838) (Acari:Psoroptidae) : diagnóstico, aspectos epidemiológicos e clínicos. 2004. 49 f. Dissertação (Mestrado) - Instituto de Veterinária - Universidade Federal Rural do Rio de Janeiro, 2004. SOUZA, C.P.; CORREIA, T.R.; MELO, R.M.P.S.; VEROCAI, G.G.; CASTRO, D.S.E.; CAVALCANTI, M.C.H.; SCOTT, F.B. Eficácia acaricida do Tiabendazol sobre Otodectes cynotis (Hering 1838) em cães. Ver. Bras Parasitol Vet., v. 15, n. 4, p.143-146, 2006.

TULESKI, G.L.R. Avaliação da prevalência infecciosa e da sensibilidade in vitro aos antimicrobianos em otites de cães. 2007.149f. Dissertação (Mestrado) - Faculdade de Medicina Veterinária, Universidade Federal do Paraná, Curitiba, 2007.

WALTON, S.; MCKINNON, M.; PIZZUTTO S.; DOUGALL, A.; WILLIAMS, E.; CURRIE, B.J. Acaricidal activity of Melaleuca alternifolia (tea tree) oil: in vitro sensitivity of sarcoptes scabiei var hominis to terpinen-4-ol. Arch. Dermatol, v. 140, n. 5, p. 563-566 2004. 